\title{
RELATIVELY OPEN MAPPINGS
}

\author{
WOO YOUNG LEE \\ (Communicated by John B. Conway)
}

\begin{abstract}
A bounded linear operator on a Banach space which is one-one, dense and 'relatively almost open' must be invertible.
\end{abstract}

\section{INTRODUCTION}

In Berberian's book [1] it is shown that, on a reflexive Banach space $X$, a non-invertible operator $T \in L(X)$ which is neither a left nor a right zerodivisor must be both a topological left and a topological right zero-divisor in the Banach algebra $L(X)$ ([1, Corollary 57.11]). In this note we show that the space $X$ need not be reflexive, and further strengthen the statement.

If $T: X \rightarrow Y$ is a bounded linear operator between normed spaces write $T^{\wedge}: X \rightarrow T(X)$ for its 'truncation': thus $T^{\wedge}$ is automatically onto, and

$$
T \text { one-one } \Longleftrightarrow T^{\wedge} \text { one-one }
$$

and

$$
T \text { bounded below } \Longleftrightarrow T^{\wedge} \text { bounded below. }
$$

We may refer the reader to [1], [2] or [3] for the concepts of "bounded below", "open" and "almost open": we shall call $T \in L(X, Y)$ relatively open if $T^{-}$ is open and call $T$ relatively almost open if $T^{\wedge}$ is almost open. The second of these concepts can be expressed in terms of the first, via duality; writing $T^{*}: Y^{*} \rightarrow X^{*}$ for the dual or adjoint of $T: X \rightarrow Y$, we have

Theorem 1. If $T \in L(X, Y)$ is a bounded linear operator between normed spaces then

$$
T \text { relatively almost open } \Longleftrightarrow T^{*} \text { relatively open. }
$$

Proof. We have, by the definition of "relatively almost open" and [2,(2.3.4)], $T$ relatively almost open $\Longleftrightarrow T^{\wedge}$ almost open $\Longleftrightarrow\left(T^{\wedge}\right)^{*}$ bounded below and, by the definition of "relatively open",

$$
T^{*} \text { relatively open } \Longleftrightarrow\left(T^{*}\right)^{\wedge} \text { open; }
$$

Received by the editors June 14, 1988.

1980 Mathematics Subject Classification (1985 Revision). Primary 47A99; Secondary 46B99. 
if $\left(T^{\wedge}\right)^{*}$ is bounded below then $\left\|g^{\circ}\right\|_{T(X)^{*}} \leq k\left\|g^{\circ} T\right\|_{X^{*}}$ for all $g^{\circ} \in T(X)^{*}$; now for arbitrary $f=g T \in T^{*}\left(Y^{*}\right)$ use the Hahn-Banach Theorem to extend the restriction $g^{\circ}$ of $g$ to $T(X)$ to $h \in Y^{*}$ with $\|h\|_{Y^{*}}=\left\|g^{\circ}\right\|_{T(X)^{*}}$ to obtain $f=g T \in\left\{h T:\|h\|_{Y^{*}} \leq k\|f\|_{X^{*}}\right\}$, which says that $\left(T^{*}\right)^{\wedge}$ is open. Conversely if this is assumed then $\|g\|_{T(X)^{*}}=\|h\|_{T(X)^{*}} \leq\|h\|_{Y^{*}} \leq k\|g T\|_{X^{*}}$.

Our main result is

Theorem 2. If $T \in L(X)$ for a Banach space $X$ then

$T$ one-one, dense and relatively almost open $\Longrightarrow T$ invertible.

Proof. Since

$$
T \text { dense } \Longleftrightarrow T^{*} \text { one-one }
$$

we have, by (1.1),

$T$ dense and relatively almost open $\Longrightarrow T^{*}$ one-one and relatively open, which means $T^{*}$ is bounded below; thus $T$ is almost open (cf. [2, (2.3.4)]); quoting half the open mapping theorem ([4, Lemma 5.2.2]) we can conclude that $T$ is open.

To see how this relates to Berberian's result, recall that the divisors of zero in the Banach algebra $L(X)$ are the operators which are not one-one, or not dense, while the topological zero-divisors are those which are not bounded below, or not almost open:

Theorem 3. If $T \in L(X)$ is not relatively almost open then it is both a topological left and a topological right zero-divisor.

Proof. If $T$ is not relatively almost open then it is not almost open, and it is not relatively open, therefore not bounded below.

The converse of Theorem 3 fails (consider the operator 0 ).

\section{ACKNOWLEDGMENT}

I wish to express my appreciation to the referee whose remarks and observations led to an improvement of the paper.

\section{REFERENCES}

1. S. K. Berberian, Lectures in functional analysis and operator theory, Springer-Verlag, New York, 1974.

2. R. E. Harte, Almost open mappings between normed spaces, Proc. Amer. Math. Soc. 90 (1984) 243-249.

3. $\ldots$, Invertibility and singularity for bounded linear operators, Marcel Dekker, New York, 1988.

4. A. Wilansky, Modern methods in topological vector spaces, McGraw-Hill, New York, 1978.

Department of Mathematics, Sung Kyun Kwan University, Seoul 110-745, Korea 